\title{
Podocin, a raft-associated component of the glomerular slit diaphragm, interacts with CD2AP and nephrin
}

\author{
Karin Schwarz, ${ }^{1}$ Matias Simons, ${ }^{1}$ Jochen Reiser, ${ }^{1}$ Moin A. Saleem, ${ }^{2}$ Christian Faul, ${ }^{1}$ \\ Wihelm Kriz, ${ }^{3}$ Andrey S. Shaw, ${ }^{4}$ Lawrence B. Holzman, ${ }^{5}$ and Peter Mundel ${ }^{1}$ \\ ${ }^{1}$ Department of Medicine and Department of Anatomy and Cell Biology, Albert Einstein College of Medicine, \\ Bronx, New York, USA \\ ${ }^{2}$ Children's Renal Unit and Academic Renal Unit, University of Bristol, Southmead Hospital, Bristol, United Kingdom \\ ${ }^{3}$ Department of Anatomy and Cell Biology, University of Heidelberg, Heidelberg, Germany \\ ${ }^{4}$ Department of Pathology and Immunology, Washington University School of Medicine, St. Louis, Missouri, USA \\ ${ }^{5}$ Department of Internal Medicine, Division of Nephrology, University of Michigan, Ann Arbor, Michigan, USA \\ Address correspondence to: Peter Mundel, Division of Nephrology, Albert Einstein College of Medicine, \\ 1300 Morris Park Avenue, Bronx, New York 10461, USA. \\ Phone: (718) 430-3219; Fax: (718) 430-8963; E-mail: mundel@aecom.yu.edu.
}

Received for publication March 30, 2001, and accepted in revised form October 11, 2001.

\begin{abstract}
NPHS2 was recently identified as a gene whose mutations cause autosomal recessive steroid-resistant nephrotic syndrome. Its product, podocin, is a new member of the stomatin family, which consists of hairpin-like integral membrane proteins with intracellular $\mathrm{NH}_{2}$ - and $\mathrm{COOH}$-termini. Podocin is expressed in glomerular podocytes, but its subcellular distribution and interaction with other proteins are unknown. Here we show, by immunoelectron microscopy, that podocin localizes to the podocyte foot process membrane, at the insertion site of the slit diaphragm. Podocin accumulates in an oligomeric form in lipid rafts of the slit diaphragm. Moreover, GST pull-down experiments reveal that podocin associates via its $\mathrm{COOH}$-terminal domain with $\mathrm{CD} 2 \mathrm{AP}$, a cytoplasmic binding partner of nephrin, and with nephrin itself. That podocin interacts with CD2AP and nephrin in vivo is shown by coimmunoprecipitation of these proteins from glomerular extracts. Furthermore, in vitro studies reveal direct interaction of podocin and CD2AP. Hence, as with the erythrocyte lipid raft protein stomatin, podocin is present in high-order oligomers and may serve a scaffolding function. We postulate that podocin serves in the structural organization of the slit diaphragm and the regulation of its filtration function.

J. Clin. Invest. 108:1621-1629 (2001). DOI:10.1172/JCI200112849.
\end{abstract}

\section{Introduction}

Glomerular podocytes are highly specialized cells consisting of a cell body, major processes, and foot processes with their interconnecting slit diaphragms (SDs) (1). As part of the glomerular filtration barrier, the $\mathrm{SD}$ is thought to function as a size-selective filter, whereas the charge selectivity is thought to be located in the glomerular basement membrane $(2,3)$. Under normal conditions, the filtration barrier is freely permeable to water, ions, and proteins smaller than albumin. In the nephrotic syndrome, the normal podocyte substructure is lost, with effacement of podocyte foot processes and massive proteinuria (4, 5). Although relatively little is known about the cellular or molecular changes that occur within podocytes during the development of nephrotic syndrome, cytoskeletal proteins likely play a central role in these changes (4). Indeed, mutations in ACTN4, encoding $\alpha$-actinin-4, cause familial focal segmental glomerulosclerosis (FSGS) (6). Due to the central role of podocytes in glomerular physiology and pathology, various groups have investigated podocyte proteins including CD2AP and nephrin. Mutations in nephrin lead to congenital nephrotic syndrome in humans (7-11), and deletion of CD2AP leads to experimental congenital nephrotic syndrome $(12,13)$.

Recently, NPHS2 was identified by positional cloning to be the target gene of autosomal recessive steroidresistant nephrotic syndrome (14). The NPHS2 gene product, podocin, is a new member of the band-7stomatin protein family of lipid raft-associated proteins (15). Comparison of amino acid sequence demonstrates that podocin is $47 \%$ identical to human stomatin and $44 \%$ to Caenorhabditis elegans Mec-2 (14, 16). Due to its structural similarity to stomatin, podocin is predicted to be an integral membrane protein with both $\mathrm{NH}_{2}$ - and $\mathrm{COOH}$-terminal intracellular domains that form a hairpin-like structure $(14,17,18)$. Podocin expression is restricted to podocytes as shown by in situ RNA hybridization (14), but its subcellular distribution is unknown. Mutations in NPHS2 are associated with familial steroid-resistant nephrotic syndrome manifest as early childhood onset of proteinuria, rapid progression to end-stage renal disease, and FSGS. This suggests a regulatory role for podocin in determining glomerular permeability. 
In the present study, we asked whether podocin acts directly at the filtration barrier and/or via interaction with other components of the SD such as nephrin or CD2AP. Using a polyclonal antibody, podocin was localized at the insertion site of the SD in podocyte foot processes. Podocin and, in part, nephrin and CD2AP are enriched in Triton X-100-insoluble lipid microdomains. We show that podocin forms highorder oligomers and that the COOH-terminal cytoplasmic domain of podocin interacts in vivo with CD2AP and nephrin. Furthermore, by in vitro studies we show a direct interaction of podocin and CD2AP. We propose that podocin acts as a scaffold protein required to maintain or regulate the structural integrity of the SD.

\section{Methods}

cDNA cloning and sequencing. Database searches with the human podocin cDNA sequence identified one EST clone (GenBank: AW106985; Research Genetics, Huntsville, Alabama, USA), which contained the complete open reading frame of mouse podocin. The full-length mouse podocin cDNA was also cloned by RT-PCR from mouse glomerular RNA. Sequence alignments, analyses, and database searches were done with the software program package HUSAR (Heidelberg Unix Sequence Analysis Resources; German Cancer Research Center, Heidelberg, Germany) as previously described (19).

Cloning and expression of podocin-GST fusion proteins. The $\mathrm{NH}_{2}$-terminus (amino acids 1-105) and the COOH-terminus (amino acids 125-385) of podocin were generated by PCR and cloned in frame into a modified PGEX vector (kindly provided by Ben Margolis) using the restriction sites EcoRI and SalI. The $\mathrm{NH}_{2}$-terminus of podocin was amplified using the primers pGSTagN-5 (AATTGAATTCTTATGATGGACTTTTTTGCGCGGA) and pGSTagN-3 (TAATGTCGACTAATCCAGAGGGCTTGATGCC). For the COOH-terminus the primers pGSTagC5 (AATTGAATTCTTATGGAGATAGACGCTGTCTGCTAC) and pGSTagC-3 (TAATGTCGACCTATAACATAGGAGAGTCCTTC) were used. Restriction sites are underlined. Resulting constructs were sequenced to confirm in-frame cloning and the absence of mutations. Fusion proteins were expressed in Escherichia coli Bl21 (Stratagene, La Jolla, California, USA) at $30^{\circ} \mathrm{C}$. Bacteria were cultivated in LB medium supplemented with $2 \%$ glucose to an $\mathrm{OD}_{600}=0.8$ and, in the case of the podocin $\mathrm{COOH}$-terminal fragment, in the presence of the proteasome inhibitor ALLN (50 $\mu \mathrm{m}$; Sigma-Aldrich, St. Louis, Missouri, USA). GST fusion proteins were induced with $1 \mathrm{mM}$ isopropyl- $\beta$-d-thiogalactopyronoside. Cells were pelleted at 3,000 $\mathrm{g}$ for 10 minutes at $4^{\circ} \mathrm{C}$, and pellets were resuspended in lysis buffer (PBS containing $2 \%$ Triton X-100, $1 \mathrm{mg} / \mathrm{ml}$ lysozyme, and protease inhibitors). After one freeze-thaw cycle the bacteria were sonicated on ice and cleared from cell debris by centrifugation at $13,000 \mathrm{~g}$ for 30 minutes at $4^{\circ} \mathrm{C}$. The efficiency of bacterial lysis was checked by
SDS-PAGE and Western blotting. The supernatants were purified on prepacked fast-flow Gluthathione Sepahrose 4B columns (Sigma-Aldrich) according to the manufacturer's instructions.

Generation of polyclonal antibodies against podocin. Rabbits were immunized with a keyhole limpet hemocyanin-conjugated peptide (single letter code: SKPVEPLNPKKKDSPML) corresponding to the COOHterminus of mouse and human podocin, which are $100 \%$ identical in this region of the molecule (box in Figure 1a). The antiserum was affinity-purified with the corresponding peptide linked to Ultralink (Pierce Chemical Co., Rockford, Illinois, USA) according to the manufacturer's instructions.

Protein extraction and immunoblotting. For protein extraction, glomeruli of adult C57 black mice were isolated as described before (19). Isolated glomeruli were pelleted by centrifugation $\left(1,000 \mathrm{~g}, 4^{\circ} \mathrm{C}, 5\right.$ minutes) and resuspended in 5 volumes of homogenization buffer (20 mM Tris, 0.5\% NP-40, 150 mM NaCl, pH 7.5) supplemented with protease inhibitors. Protein extraction was carried out at $4^{\circ} \mathrm{C}$ using 15 strokes in a Dounce homogenizer, and insoluble material was pelleted at $14,000 \mathrm{~g}$ for 10 minutes at $4^{\circ} \mathrm{C}$. The resulting pellet was resuspended in 5 volumes of homogenization buffer and processed as above. Homogenates from both steps were pooled and passed 20 times through a $27 \mathrm{G}$ needle. SDS-PAGE and Western blotting were done essentially as described before (19) with the modification that in some experiments reducing agents were avoided in the sample buffer. The affinity-purified primary antibody against podocin was used at 1:250 and 1:500. The polyclonal antibody against the intracellular domain of nephrin (20) was used at 1:1,000, polyclonal antiCD2AP (12) at 1:2,500, and polyclonal anti-caveolin-1 (kindly provided by Michael P. Lisanti) at 1:1,000. The monoclonal anti-human transferrin receptor antibody (Zymed Laboratories Inc., South San Francisco, California, USA) was used at 1:800, the monoclonal anti- $\alpha$ tubulin (Oncogene Research Products, Cambridge, Massachusetts, USA) at 1:1,000. The lamin antibody (clone X 167; Progen, Heidelberg, Germany) was used at $1: 250$. The immunoreaction was visualized using ECL substrate (Amersham Pharmacia, Piscataway, New Jersey, USA) and film exposure.

GST pull-down experiments. Bacterial lysates were prepared as described above and applied to prepacked Glutathione Sepharose 4B columns, washed with 10 bed volumes of lysis buffer (without detergent and lysozyme), and equilibrated with 5 bed volumes of coimmunoprecipitation (Co-IP) buffer (50 mM Tris, $\mathrm{pH}$ 7.5, $100 \mathrm{mM} \mathrm{NaCl}, 15$ mM EGTA, 0.1\% Triton X-100, 1 $\mathrm{mM}$ DTT, and protease inhibitors). Protein extracts from isolated glomeruli were diluted 1:2 in Co-IP buffer and applied to the GST columns as described for the bacterial lysates. Columns were washed with 20 bed volumes of Co-IP buffer, before the bound fusion protein was eluted with 4 volumes of elution buffer (EB: 10 $\mathrm{mM}$ reduced glutathione in $50 \mathrm{mM}$ Tris, $\mathrm{pH} 8.0)$. One- 
milliliter fractions were collected and aliquots were analyzed by Western blotting.

Coimmunoprecipitation. Protein extracts were prepared as described above with the addition of $1 \mathrm{mM}$ vanadate in the homogenization buffer. For each immunoprecipitation, $0.5 \mathrm{mg}$ protein extract was precleared with protein A-Sepharose 4B (Sigma-Aldrich) in $0.5 \mathrm{ml} \mathrm{TNE}$ buffer $(250 \mathrm{mM} \mathrm{NaCl}, 5 \mathrm{mM}$ EDTA, $10 \mathrm{mM}$ Tris, $\mathrm{pH}$ 7.4, proteinase inhibitors) for 1 hour at $4^{\circ} \mathrm{C}$ to remove immunoglobulin-like proteins that may bind nonspecifically to the beads. In some experiments the precleared extracts were immunoprecipitated with antinephrin, anti-CD2AP, anti-podocin, or anti-lamin antibodies cross-linked to protein A-Sepharose beads using the Seize X Protein A immunoprecipitation kit (Pierce Chemical Co.) according to the manufacturer's protocol. After addition of sample buffer without reducing agents ( $\beta$-mercaptoethanol; $\beta$-ME), samples were boiled for 3 minutes at $60^{\circ} \mathrm{C}$ and proteins were analyzed by Western blot. In other experiments, extracts were incubated with precipitating antibodies for 2 hours at $4^{\circ} \mathrm{C}$. Nonspecific aggregates were pelleted by centrifuging for 5 minutes at $14,000 \mathrm{~g}$ at $4^{\circ} \mathrm{C}$. A mixture of protein A/G-Sepharose 4B beads (SigmaAldrich) was added to the supernatants and incubated for 90 minutes at $4^{\circ} \mathrm{C}$. In this step protein $\mathrm{G}$ was added because of its broader affinity spectrum for different subtypes of antibodies compared with protein $\mathrm{A}$. The beads were washed five times in TNE buffer before bound proteins were eluted by boiling the samples for 5 minutes in sample buffer (containing $\beta$-ME) at $95^{\circ} \mathrm{C}$.

In vitro translation assays. For the in vitro translation and precipitation studies, $1 \mu \mathrm{g}$ of myc-tagged CD2AP, $1 \mu \mathrm{g}$ of podocin cDNA, or $1 \mu \mathrm{g}$ of a myc-control plasmid (constitutively active Notch3) was transcribed and translated using the TNT coupled reticulocyte lysate system (Promega Corp., Madison, Wisconsin, USA) according to the manufacturer's protocol in the presence or absence of $\mathrm{S}^{35}$-methionine. Two microliters of each reaction was analyzed separately by SDS-PAGE and autoradiography. For coimmunoprecipitations, 10 $\mu \mathrm{l}$ of $\mathrm{S}^{35}$-labeled podocin and $10 \mu \mathrm{l}$ of unlabeled CD2AP were mixed together and the volume was adjusted to $250 \mu \mathrm{l}$ by addition of TNE buffer. After 3 hours' incubation on ice, $4 \mu \mathrm{l}$ of anti-myc antibody was added and samples were incubated as described above for the Co-IP experiments. After precipitation, proteins were eluted with $100 \mu 100 \mathrm{mM}$ glycine ( $\mathrm{pH}$ 2.7) and samples were analyzed by SDS-PAGE and autoradiography. To show the specificity of the interaction, in

\section{a}

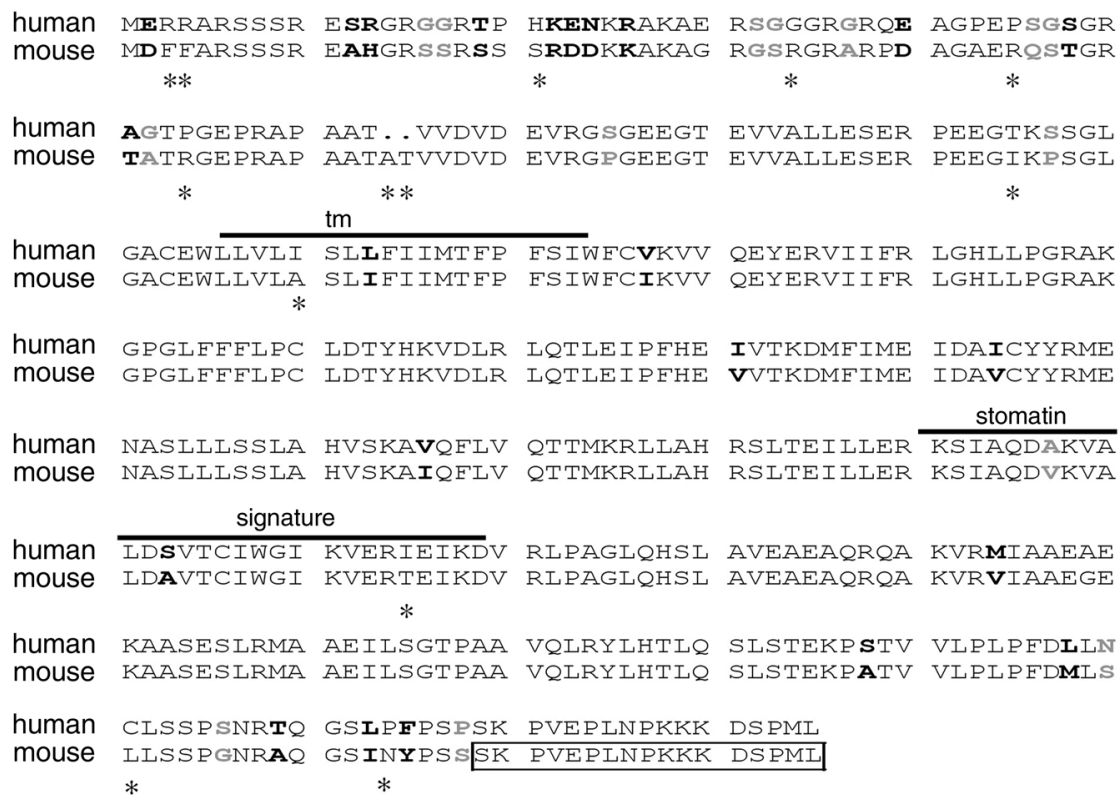

b

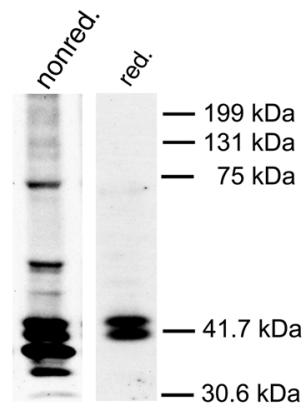

C

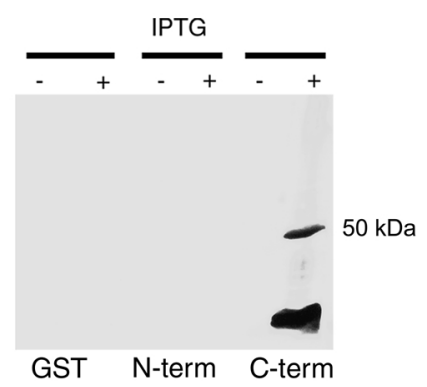

\section{Figure 1}

Molecular cloning and Western blot analysis of mouse podocin. (a) Amino acid sequence comparison of human and mouse podocin. Similar residues are shown in boldface, strong similarity in black, and weaker similarity in gray. Different amino acids are marked by asterisks. The transmembrane domain (tm) and the stomatin signature are overlined. The box indicates the peptide used for antibody generation. Most of the amino acid exchanges between human and mouse are restricted to the $\mathrm{NH}_{2}$-terminal part of the protein, whereas the $\mathrm{tm}$ domain, the stomatin signature, and the $\mathrm{COOH}$-terminal part are almost identical. The sequence data of mouse podocin are available from GenBank/EMBL/DDBJ under accession no. AJ302048. (b) Western blot analysis of glomerular extracts revealed a $42-\mathrm{kDa}$ band under reducing (red.) conditions (right lane). Under nonreducing (nonred.) conditions (left panel), additional higher molecular bands appeared, indicating podocin dimerization. (c) The specificity of the podocin antibody was confirmed by Western blot analysis of recombinant GST-podocin fragments and GST alone. Only the $\mathrm{COOH}$-terminal fusion protein (C-term) was recognized by the antibody. $\mathrm{N}$-term, $\mathrm{NH}_{2}$-terminal fusion protein. 


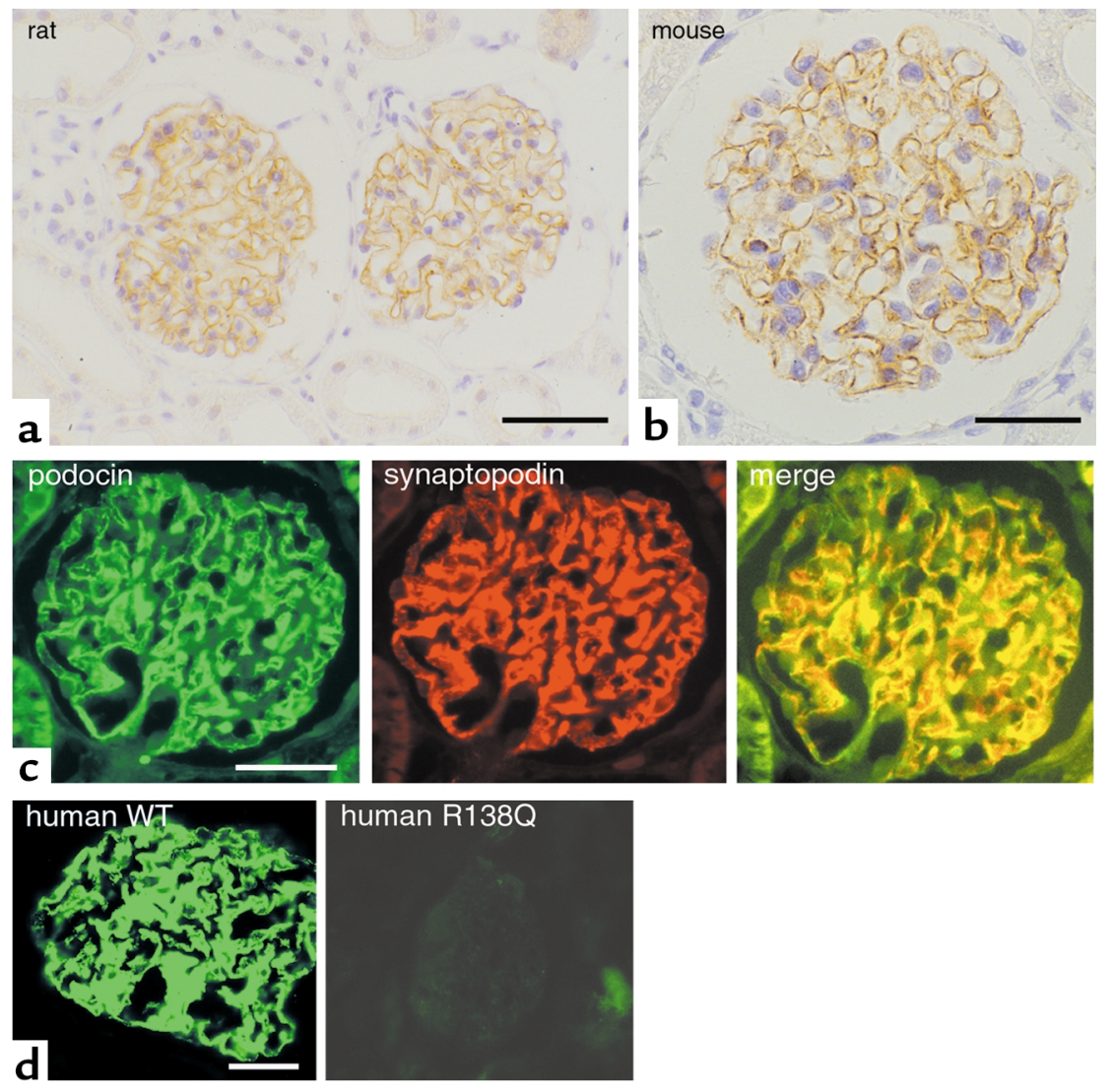

\section{Figure 2}

Expression of podocin in kidney tissues. Immunostaining of rat (a) and mouse (b) adult kidney sections showing the glomerular expression of podocin. (c) Double labeling of podocin (green) with the podocyte foot process marker synaptopodin (red) results in a complete overlap of both signals (yellow in merge). (d) Expression pattern of podocin in normal human kidney (left panel) and in the kidney of a patient with steroid-resistant nephrotic syndrome (right panel). The patient belongs to the group of patients originally described by Boute and coworkers (14). Interestingly, podocin expression can be detected only in the normal kidney, but not in the kidney of the patient with the podocin mutation. WT, wild-type. Bars: a, $30 \mu \mathrm{m}$; b, $15 \mu \mathrm{m}$; c, 20 $\mu \mathrm{m} ; \mathbf{d}, 25 \mu \mathrm{m}$. vitro translated radioactive-labeled podocin was incubated either with the anti-myc antibody alone or in the presence of the myc-tagged control protein. For the GST pull-down experiments the podocin fragments and GST were expressed and isolated as described above. One hundred microliters of the respective fusion protein bound to Sepharose beads was incubated with $30 \mu \mathrm{l}$ of radioactive-labeled CD2AP in a final volume of $250 \mu \mathrm{l}$ Co-IP buffer overnight at $4^{\circ} \mathrm{C}$. Samples were washed with Co-IP buffer until only background levels of radioactivity were detectable in the GST sample. Bound proteins were eluated with EB and analyzed by SDS-PAGE and autoradiography.

Sucrose gradient ultracentrifugation. For preparation of low-density Triton X-100-insoluble membranes (detergent-resistant membranes [DRMs]), isolated glomeruli from eight adult mice were homogenized by 14 strokes in a Dounce homogenizer in $1 \mathrm{ml}$ TNE buffer $(250 \mathrm{mM} \mathrm{NaCl}, 5 \mathrm{mM}$ EDTA, $10 \mathrm{mM}$ Tris, $\mathrm{pH}$ 7.4 , proteinase inhibitors). Insoluble material was pelleted at $14,000 \mathrm{~g}$ for 10 minutes at $4^{\circ} \mathrm{C}$. The resulting pellet was resuspended in $1 \mathrm{ml} \mathrm{TNE}$ and processed as above. Homogenates from both steps were pooled and passed 20 times through a $27 \mathrm{G}$ needle. The optimal Triton X-100/protein ratio for the preparation of DRMs was established in pilot studies, using $0.2 \%$, $0.5 \%$, and $1 \%$ Triton X-100 (data not shown). These gradients were tested for intact rafts using caveolin-1 as positive marker for DRM fractions and transferrin receptor as negative marker to show that these rafts were free of contaminating non-DRM membranes. In these experiments $0.2 \%$ Triton $\mathrm{X}-100$ showed the best results in isolating DRMs from freshly isolated glomeruli and was therefore used for all experiments. The lysates were incubated for 45 minutes on ice in the presence of $0.2 \%$ Triton X-100 and brought to $40 \%$ sucrose. Samples were then overlaid with a sucrose step gradient $(5 \mathrm{ml}$ of $35 \%$ sucrose, $1 \mathrm{ml}$ of $15 \%$ sucrose, $1 \mathrm{ml}$ of $5 \%$ sucrose, $1 \mathrm{ml} \mathrm{TNE}$ ) as described by Mora et al. (21). Gradients were centrifuged for 20 hours at $120,000 \mathrm{~g}$ at $4^{\circ} \mathrm{C}$ in a swing-out rotor, and 12 fractions ( $1 \mathrm{ml}$ each) were collected starting from the top and analyzed by SDS-PAGE.

Velocity gradient centrifugation. The oligomerization capacity of podocin, CD2AP, and nephrin was analyzed by velocity gradient centrifugation in sucrose performed essentially as described by Zaliauskiene et al. (22). Discontinuous 5-60\% (wt/wt) sucrose gradients were laid over an $80 \%$ sucrose cushion $(300 \mu \mathrm{l})$ in SW-60 tubes. Sucrose percentage difference between the fractions was 4\%. Glomerular extracts were prepared in MNT buffer (100 mM NaCl, 20 mM Tris, 30 mM 2-(N-morpholino)ethansulfonic acid, $\mathrm{pH} 5.8$ ) containing $1 \%$ Triton $\mathrm{X}$ 100 as described above. After centrifugation, 13 fractions $(350 \mu \mathrm{l}$ each) were collected starting from the top and analyzed for oligomeric proteins by Western blot.

Immunohistochemistry and immunoelectron microscopy. Immunoperoxidase histochemistry of $3-\mu \mathrm{m}$-thick seri- 
al sections of formalin-fixed paraffin-embedded tissue was done as described before (23). Immunofluorescence labeling of paraffin-embedded mouse kidney sections was done as previously described (19). Human frozen samples were processed as described for the mouse sections. Immunogold labeling of ultrathin frozen sections of perfusion-fixed rat and mouse kidney with the affinity-purified anti-podocin antiserum was done as previously described (24). The sections were observed under a Phillips EM 301 electron microscope (Phillips, Eindhoven, The Netherlands).

\section{Results}

Molecular cloning reveals bigh sequence similarity between mouse and human podocin. The mouse podocin cDNA was cloned from glomerular RNA and a mouse kidney EST clone. The predicted full-length open reading frame encodes a 385-amino acid protein with a calculated molecular mass of $42 \mathrm{kDa}$ (Figure 1a). Sequence alignments with human podocin revealed $86 \%$ identity and an overall similarity of $89 \%$ at the amino acid level (Figure 1a). Interestingly, most of the amino acid substitutions between the two species (asterisks in Figure 1a) were restricted to the $\mathrm{NH}_{2}$-terminus, whereas the transmembrane domain, the stomatin signature, and the $\mathrm{COOH}$-terminus were virtually identical.

Generation and characterization of polyclonal antibodies against podocin. Using a rabbit polyclonal antiserum directed against the last 15 amino acids of the $\mathrm{COOH}$ terminus of mouse and human podocin, a protein with an apparent molecular mass of $42 \mathrm{kDa}$ was recognized by Western blot analysis of isolated mouse glomeruli under reducing conditions (Figure $1 \mathrm{~b}$, right lane). This is consistent with the predicted molecular mass. Interestingly, we detected a double band for podocin. The upper band most likely represents a posttranslational modified variant of podocin, since it was not seen when using the podocin CDNA as template for in vitro translation (see Figure 5c, left panel). Under nonreducing conditions additional bands of higher molecular weight were detected in the same extract (Figure 1b, left lane), suggesting the presence of podocin oligomers. To test the specificity of the antibody, we expressed the $\mathrm{NH}_{2}-$ terminal part of podocin or the $\mathrm{COOH}$-terminal part as GST fusion proteins and performed Western blot analysis with the affinity-purified anti-podocin antibody. Only the $\mathrm{COOH}$-terminal fusion protein containing the peptide sequence used for immunization was recognized by the antibody (Figure 1c). By immunofluorescence microscopy, the antibody stained normal human kidney but no signal was found in a kidney biopsy from a patient with steroid-resistant nephrotic syndrome (Figure 2d). Hence, we conclude that the antibody is specific for the $\mathrm{COOH}$-terminus of podocin.

Immunohistochemical detection of podocin in podocytes of adult rat and mouse kidneys. Using immunohistochemistry, we observed strong staining in glomeruli of rat (Figure 2a) and mouse (Figure 2b) kidneys. No signal was detected in other parts of the kidney. Double label- ing immunofluorescence studies on paraffin-embedded sections of adult mouse kidney were performed using anti-synaptopodin (19) as an established specific marker of podocyte foot processes, and anti-podocin. These studies revealed a complete overlap of podocin and synaptopodin expression in the glomerulus (Figure 2c). Hence podocin expression is restricted to podocytes.

Podocin is a cell membrane protein localized at the insertion site of the SD complex in podocyte foot processes. The precise subcellular localization of podocin was determined by immunogold labeling of ultrathin frozen sections from rat and mouse kidney cortex. Gold particles exclusively decorated the SD region between podocyte foot processes and were not detected in other glomerular cell types (Figure 3). As shown in Figures 3, a and b, podocin was found in the vicinity of the attachment of the SD to the foot process. Thus, we confirmed the podocyte-specific expression of podocin and showed its association with the junctional complex of the SD.

Podocin, CD2AP, and nephrin associate with lipid rafts. Since podocin contains a putative transmembrane domain and is homologous with the lipid raft-associated protein stomatin (25), we asked whether podocin might also be raft-associated. In flotation gradients of Triton X-100 extracts, raft-associated proteins float to the top as DRM fractions, whereas detergent-soluble proteins or detergent-insoluble protein complexes that associate with the cytoskeleton are identified as higher-density fractions (26). As shown in Figure 4a, most of the podocin was found in fraction 3 , which contains the DRMs. The quality and purity of the fractions were
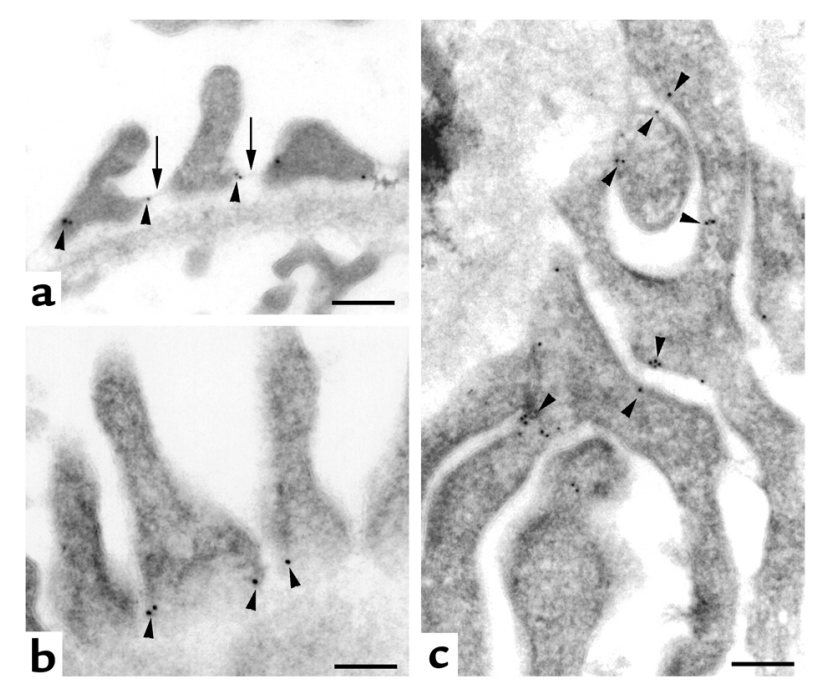

\section{Figure 3}

Podocin localizes to the SD. Immunogold labeling shows the subcellular distribution of podocin (arrowheads) at the SD (arrows). (a and b) Cross sections of adult rat kidney. (c) A low-power magnification of a flat section. The gold particles are specifically located at the insertion site of the SD in the podocyte cell membrane (arrows in a), whereas no label can be detected in the glomerular basement membrane. $\mathbf{b}$ shows a high-power magnification of $\mathbf{a}$. In $\mathbf{c}$, the tangential section shows exclusive labeling of podocyte foot processes (arrowheads). Bars: $\mathbf{a}$ and c, $100 \mathrm{~nm}$; b, $200 \mathrm{~nm}$. 


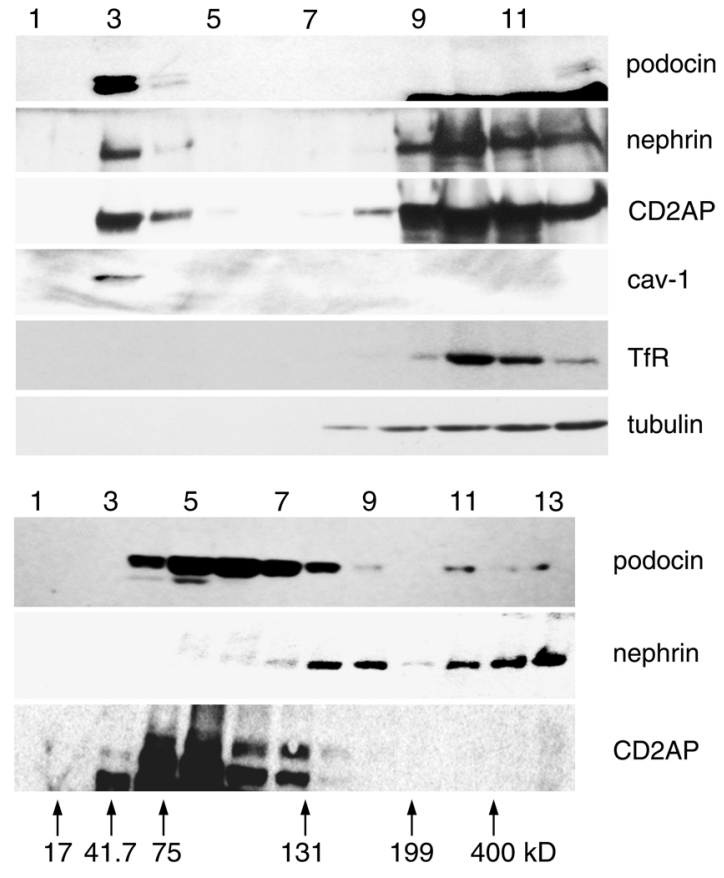

Figure 4

Podocin interacts with nephrin and CD2AP in lipid rafts. (a) Flotation analysis of podocin, nephrin, and CD2AP in a sucrose step gradient. The purity of the fractions was confirmed using caveolin-1 (cav-1) as raft marker and transferrin receptor (TfR) showing the absence of contaminating nonraft membranes in the DRM fraction (lane 3). Podocin is preferentially found in the DRM fraction (lane 3 ), whereas nephrin and CD2AP are found in the DRM fraction and the high sucrose fractions (lanes 9 and 10). The cytosolic protein tubulin is exclusively found in the heavy fractions (lanes 8-12). (b) The ability of podocin, nephrin, and CD2AP to form oligomers was tested by velocity gradient centrifugation. Arrows indicate molecular weight markers. In contrast to CD2AP, both nephrin and podocin were found to form high-order complexes.

assessed using caveolin- 1 as a raft marker, the exclusion of the transferrin receptor as marker for non-raft-associated membrane proteins, and exclusion of tubulin to show that raft fractions were not contaminated with cytoplasmic components (Figure 4a). The transmembrane protein nephrin was found in the DRMs, but also in the heavy fractions (Figure 4a) (27). CD2AP was found to a large extent in the high-density fractions but was also detected in the DRM fraction (Figure 4a).

When evaluated by velocity gradient centrifugation, a fraction of podocin was found in gradient fractions separating with proteins of apparent molecular mass greater than $200 \mathrm{kDa}$ (Figure 4b). Hence, like stomatin (18) and caveolin-1 $(21,25,28)$, podocin forms highorder complexes. Consistent with previous results (27), we also noted oligomerization of nephrin (Figure 4b). In contrast, $\mathrm{CD} 2 \mathrm{AP}$ did not form complexes (Figure $4 \mathrm{~b}$ ).

The COOH-terminus of podocin interacts with CD2AP and nephrin. Next, we performed GST pull-down and coimmunoprecipitation studies to test whether podocin interacts with CD2AP or nephrin or both. When glomerular extract was passed over GST columns, the
$\mathrm{COOH}$-terminal fragment of podocin, but not the $\mathrm{NH}_{2}-$ terminal fragment or GST alone, specifically interacted with nephrin and CD2AP (Figure 5a). These findings are consistent with sequence data (Figure 1a) showing that the $\mathrm{COOH}$-terminus of podocin is enriched in proline residues that may act as protein-protein interaction domains. To confirm these interactions, we used antibodies against podocin, nephrin, and CD2AP to coimmunoprecipitate protein complexes from isolated glomeruli extracts of adult mice. All three antibodies immunoprecipitated podocin-containing protein complexes (Figure 5b, left panel), confirming the GST pulldown data. In contrast, an antibody against nuclear lamin, serving as negative control, failed to precipitate podocin (Figure $5 \mathrm{~b}$, left panel). Under reducing conditions the electrophoretic mobility of podocin on SDSPAGE is similar to that of the immunoglobulin heavy chain. For this reason, podocin-containing protein complexes were pulled down with primary antibodies irreversibly cross-linked to Sepharose beads. After precipitation, bound proteins were eluated under acidic conditions. Samples were resolved on SDS-PAGE under nonreducing conditions. Incubation of these samples with an irrelevant primary polyclonal antibody (SRIB1 against myopodin) revealed no reactivity on the membranes (Figure 5b, left panel). In contrast, after incubation with anti-podocin antibody, bands of $42 \mathrm{kDa}$ and approximately $75 \mathrm{kDa}$ were present, which corresponded to podocin and probably a podocin dimer or an otherwise modified podocin molecule (Figure $5 \mathrm{~b}$, left panel). We detected the same bands for podocin in Western blot analysis when running the extracts under nonreducing conditions (Figure 1b, left lane). Finally, we tested whether CD2AP and nephrin were present in coimmunoprecipitates performed with anti-podocin antibody. As shown in Figure 5b, both CD2AP and nephrin were present in immune complexes precipitated with anti-podocin antibody (right panel) but could not be detected when using the preimmune serum as control. These findings confirm the in vivo interaction of podocin with CD2AP and nephrin.

Podocin directly interacts with CD2AP. To investigate whether the in vivo interaction between podocin and CD2AP resulted from direct interactions, we performed in vitro translation and coimmunoprecipitation studies. Podocin and myc-CD2AP were translated in vitro using a coupled reticulocyte lysate system in the presence (Figure $5 c$, left panel) or absence of $\mathrm{S}^{35}$-labeled methionine. For Co-IPs, in vitro translated $\mathrm{S}^{35}$-labeled podocin was mixed with unlabeled CD2AP and incubated with anti-myc antibody in TNE buffer. In control experiments, $\mathrm{S}^{35}$-podocin was incubated with anti-myc antibody in the absence of myc-tagged CD2AP, with anti-myc antibody in the presence of an irrelevant myctagged control protein, or with anti-podocin antibody. As shown in Figure 5c, CD2AP and podocin directly interacted with each other (middle panel). Interestingly, after Co-IP with anti-podocin, only a podocin monomer was detected (Figure 5c), whereas in anti-myc 
precipitates following incubation of podocin with mycCD2AP, both a podocin monomer and a dimer were present (Figure $5 \mathrm{c}$ ). The interaction between podocin and $\mathrm{CD} 2 \mathrm{AP}$ was specific, since no signal was detected with the irrelevant myc-tagged protein or with the antimyc antibody alone (Figure $5 \mathrm{c}$, middle panel). Finally, the specificity of the interaction was further confirmed by GST pull-downs with GST alone, the $\mathrm{NH}_{2}$-terminal part of podocin or the $\mathrm{COOH}$-terminal part of podocin, and $\mathrm{S}^{35}$-labeled CD2AP (Figure 5c, right panel). As in the pull-down experiments from glomerular extracts (Figure 5a), only the $\mathrm{COOH}$-terminal podocin fusion protein interacted with $\mathrm{S}^{35}-\mathrm{CD} 2 \mathrm{AP}$.

\section{Discussion}

The SD complex contains at least three transmembrane proteins, nephrin $(13,20,29,30)$, P-cadherin (24), and FAT (31), that may account for its zipperlike structure. At the cytoplasmic insertion site of the SD, ZO-1 (32), $\alpha$-, $\beta$-, and $\gamma$-catenins $(24,33)$, and CD2AP (12) are present. The SD may represent a modified adherens junction (24) and is primarily responsible for the size selectivity of the glomerular filter $(2,3)$. Previous genetic studies have revealed that nephrin,
$\mathrm{CD} 2 \mathrm{AP}$, and podocin are indispensable for the normal filtration function of the SD. In this study, podocin, a novel integral membrane protein possessing a characteristic hairpin loop topology, has been identified as another component of the SD.

The assembly of the SD complex commences when podocyte precursors differentiate from typical polarized epithelial cells of the S-shaped body to more mature mesenchymal-like cells of the capillary loop stage $(20,34)$. Apical tight junctions migrate downward and convert into SDs $(32,35)$. Podocyte dedifferentiation is observed in pathological situations in humans and experimental models (35-37), suggesting dynamic regulation of the SD complex under these conditions. The temporal expression pattern of podocin during kidney development is similar to that of nephrin as shown by in situ hybridization (14). Podocin first appears in the S-shape stage in the future podocytes. The expression of podocin is maintained during later stages of podocyte development and in mature kidneys (14). CD2AP first appears in podocytes during the capillary loop stage (38). CD2AP mutant mice show no alteration of nephrin distribution during kidney development, but in 7-week-old mice, when most of the a

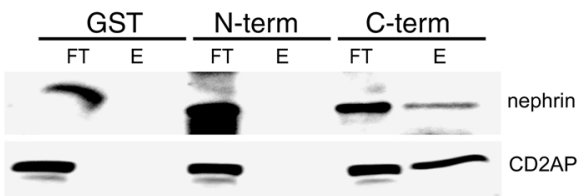

b
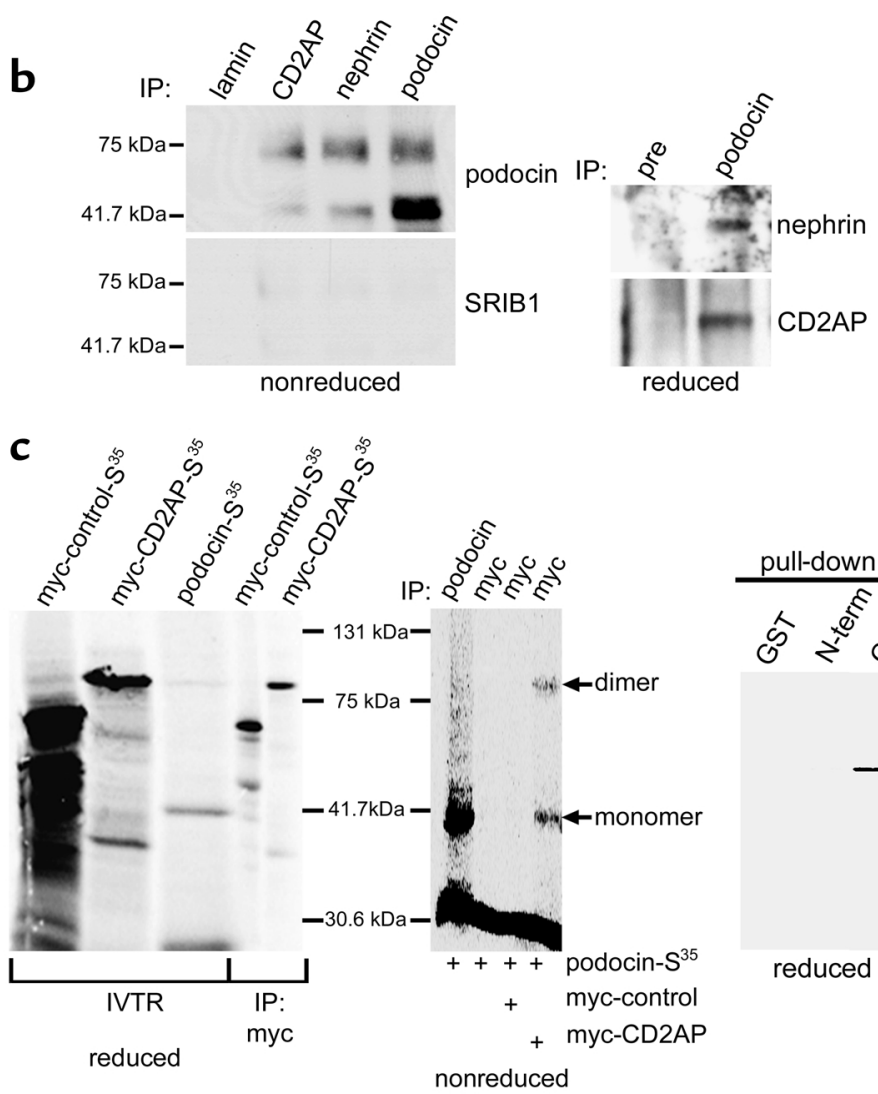

\section{Figure 5}

The $\mathrm{COOH}$-terminus of podocin interacts with nephrin and CD2AP. (a) The COOH-terminus of podocin associates with nephrin and CD2AP as shown by GST pull-downs. Nephrin and CD2AP are detectable in the final eluate (E) of the GSTpodocin- $\mathrm{COOH}$ column. GST alone and the podocin $\mathrm{NH}_{2}$-terminus do not interact with these two SD components, as indicated by detection of nephrin and CD2AP in the flow-through (FT) fraction but not in the eluate. (b) The GST pull-down data were confirmed by Co-IPs with cross-linked antibodies against podocin, nephrin, CD2AP, or lamin (negative control) from glomerular extracts. Bound proteins were eluated and analyzed by SDSPAGE and Western blot under nonreducing conditions (left panel) or in the presence of $\beta$-ME (right panel). Blots were then incubated with an irrelevant antibody (SRIB1), or with anti-podocin, antiCD2AP, or anti-nephrin antibodies. The right panel shows Co-IPs of CD2AP and nephrin with antipodocin antibody or preimmune serum (pre) under reducing conditions. (c) The ability of podocin to directly interact with CD2AP was tested by coimmunoprecipitation of in vitro translated proteins and by GST pull-downs. The left panel shows the in vitro translated $S^{35}$-labeled proteins. Co-IP of unlabeled CD2AP and $\mathrm{S}^{35}$-labeled podocin showed direct interaction of both proteins (middle panel). The specificity of the podocin-CD2AP interaction was further confirmed by pull-down experiments, using recombinant podocin-GST fragments and radioactive-labeled $\mathrm{CD} 2 \mathrm{AP}$ (right panel). Only with the $\mathrm{COOH}$-terminal part of podocin, $\mathrm{CD} 2 \mathrm{AP}$ was coprecipitated. IVTR, in vitro translation reaction. 
glomeruli are already severely damaged, nephrin is hardly detectable in the glomeruli (12). Taken together, these data and the data of the present study suggest that all three proteins, nephrin, CD2AP, and podocin, are necessary to maintain the structural integrity of the $\mathrm{SD}$. Furthermore, the normal distribution of nephrin in postnatal kidneys of CD2AP-deficient mice may be due to its interaction with podocin, since podocin and nephrin are the first proteins of this group to be expressed in podocytes during kidney development.

This study shows that podocin can form complexes and associates with lipid rafts. The observed oligomerization not only resembles the oligomerization found for nephrin (Figure 4b) (27); it may also represent a typical feature observed for other hairpin loop membrane proteins like caveolins and stomatin $(15,17,39$, $40)$. The oligomerization of caveolin clusters rafts and triggers plasma membrane invaginations termed caveolae (41). Here, we showed that podocin is associated with Triton X-100-insoluble lipid membranes and is likely to be raft-associated, forming oligomeric complexes in rafts (Figure 4b). Hence, podocin may act as a scaffolding protein in podocyte lipid rafts, recruiting nephrin and CD2AP to these microdomains. Like caveolin, the oligomerization of podocin could cluster nephrin-containing rafts (27) and thereby trigger SD assembly. The assembled SD complex could then be held together by a network of nephrin and podocin oligomers in which protein-protein interactions apart from lipid-protein interactions come into play. This study suggests that podocin interacts with nephrin via the $\mathrm{COOH}$-terminus.

Apart from an interaction between nephrin and podocin, CD2AP could serve as a linker protein at the cytoplasmic side of the plasma membrane. Our data show that CD2AP interacts directly with podocin and is in part found in the same DRM fraction as podocin and nephrin. Further detailed biochemical studies are needed to elucidate the protein relations and the stochiometry of the SD complex. Where the assembly of the SD complex occurs is an interesting question. Podocin may have a crucial role in the assembly of the complex. It is not known whether the complex assembles during its transport along the biosynthetic pathway to the plasma membrane or directly at the level of the SD. Furthermore, the presence of other proteins within the SD complex needs to be examined carefully. In particular, signaling proteins, like protein kinases and phosphatases, may be required to regulate the assembly of the complex and the integration of newly synthesized proteins. Having a high affinity for lipid rafts, kinases of the src family represent good candidates.

In summary, we have demonstrated that podocin, the target protein of autosomal recessive steroid-resistant nephrotic syndrome (14), is a novel component of the glomerular SD complex. In particular, (a) podocin is expressed in the podocyte foot process cell membrane at the insertion site of SD as shown by immuno-electron microscopy; (b) podocin, nephrin, and CD2AP are associated with lipid rafts of the SD; (c) podocin not only colocalizes but interacts with CD2AP and nephrin in vivo as shown by Co-IP studies of glomerular extracts; (d) podocin binds to CD2AP and nephrin via its $\mathrm{COOH}$-terminus and may thereby serve as scaffolding protein in the organization of the SD complex; (e) podocin can directly bind to CD2AP. Since CD2AP has recently been shown to directly interact with nephrin in vitro (12) and in vivo (42), it may serve as an adapter protein mediating the interaction of podocin and nephrin. On the other hand, podocin and nephrin could also directly interact with each other. An intact SD is essential for normal glomerular filtration, and alterations of only one of its key components, nephrin, CD2AP, or podocin, leads to massive proteinuria $(5,11-14,43)$. The results of the present study may open new avenues for understanding the underlying pathomechanisms in glomerular diseases with proteinuria.

Note added in proof. The interaction of podocin with nephrin was also found by T.B. Huber et al. (Huber, T.B., et al. 2001. Interaction with podocin facilitates nephrin signaling. J. Biol. Chem. 276:41543-41546.)

\section{Acknowledgments}

We would like to thank Torsten Heider, Hiltraud Hosser, and Bruni Haenhel for expert technical assistance. We would also like to thank Daniela Volonte and Michael P. Lisanti (Albert Einstein College of Medicine [AECOM] for advice on the raft experiments and the anti-caveolin-1 antibody and Ben Margolis (Ann Arbor, Michigan, USA) for the modified PGEX vector. We would also like to thank Barbara Ballermann (AECOM) for critical reading of the manuscript. This work was supported by grants from the Howard Hughes Medical Research Institute Research Resources for Medical Schools and NIH (DK 57683-01) to Peter Mundel. Karin Schwarz was supported by a fellowship from the National Kidney foundation of New York/New Jersey.

1. Mundel, P., and Kriz, W. 1995. Structure and function of podocytes: an update. Anat. Embryol. (Berl.) 192:385-397.

2. Daniels, B.S., Deen, W.M., Mayer, G., Meyer, T., and Hostetter, T.H. 1993. Glomerular permeability barrier in the rat. Functional assessment by in vitro methods. J. Clin. Invest. 92:929-936.

3. Drumond, M.C., and Deen, W.M. 1994. Structural determinants of glomerular hydraulic permeability. Am. J. Physiol. 266:F1-F12.

4. Smoyer, W.E., and Mundel, P. 1998. Regulation of podocyte structure during the development of nephrotic syndrome. J. Mol. Med. 76:172-183.

5. Somlo, S., and Mundel, P. 2000. Getting a foothold in nephrotic syndrome. Nat. Genet. 24:333-335.

6. Kaplan, J.M., et al. 2000. Mutations in ACTN4, encoding alpha-actinin4, cause familial focal segmental glomerulosclerosis. Nat. Genet. 24:251-256.

7. Kestila, M., et al. 1998. Positionally cloned gene for a novel glomerular protein-nephrin-is mutated in congenital nephrotic syndrome. Mol. Cell. 1:575-582.

8. Bolk, S., Puffenberger, E.G., Hudson, J., Morton, D.H., and Chakravarti, A. 1999. Elevated frequency and allelic heterogeneity of congenital nephrotic syndrome, Finnish type, in the old order Mennonites. Am.J. Hum. Genet. 65:1785-1790.

9. Beltcheva, O., Martin, P., Lenkkeri, U., and Tryggvason, K. 2001. Mutation spectrum in the nephrin gene (NPHS1) in congenital nephrotic syndrome. Hum. Mutat. 17:368-373.

10. Aya, K., Tanaka, H., and Seino, Y. 2000. Novel mutation in the nephrin gene of a Japanese patient with congenital nephrotic syndrome of the 
Finnish type. Kidney Int. 57:401-404.

11. Tryggvason, K. 1999. Unraveling the mechanisms of glomerular ultrafiltration: nephrin, a key component of the slit diaphragm. J. Am. Soc. Nephrol. 10:2440-2445.

12. Shih, N.Y., et al. 1999. Congenital nephrotic syndrome in mice lacking CD2-associated protein. Science. 286:312-315.

13. Putaala, H., Soininen, R., Kilpelainen, P., Wartiovaara, J., and Tryggvason, K. 2001. The murine nephrin gene is specifically expressed in kidney, brain and pancreas: inactivation of the gene leads to massive proteinuria and neonatal death. Hum. Mol. Genet. 10:1-8.

14. Boute, N., et al. 2000. NPHS2, encoding the glomerular protein podocin, is mutated in autosomal recessive steroid-resistant nephrotic syndrome. Nat. Genet. 24:349-354.

15. Salzer, U., and Prohaska, R. 2001. Stomatin, flotillin-1, and flotillin-2 are major integral proteins of erythrocyte lipid rafts. Blood. 97:1141-1143.

16. Huang, M., Gu, G., Ferguson, E.L., and Chalfie, M. 1995. A stomatin-like protein necessary for mechanosensation in C. elegans. Nature. 378:292-295.

17. Salzer, U., Ahorn, H., and Prohaska, R. 1993. Identification of the phosphorylation site on human erythrocyte band 7 integral membrane protein: implications for a monotopic protein structure. Biochim. Biophys. Acta. 1151:149-152.

18. Snyers, L., Umlauf, E., and Prohaska, R. 1998. Oligomeric nature of the integral membrane protein stomatin. J. Biol. Chem. 273:17221-17226.

19. Mundel, P., et al. 1997. Synaptopodin: an actin-associated protein in telencephalic dendrites and renal podocytes. J. Cell Biol. 139:193-204.

20. Holzman, L.B., et al. 1999. Nephrin localizes to the slit pore of the glomerular epithelial cell. Kidney Int. 56:1481-1491.

21. Mora, R., et al. 1999. Caveolin-2 localizes to the golgi complex but redistributes to plasma membrane, caveolae, and rafts when co-expressed with caveolin-1. J. Biol. Chem. 274:25708-25717.

22. Zaliauskiene, L., et al. 2000. Down-regulation of cell surface receptors is modulated by polar residues within the transmembrane domain. Mol. Biol. Cell. 11:2643-2655.

23. Mundel, T.M., Heid, H.W., Mahuran, D.J., Kriz, W., and Mundel, P. 1999. Ganglioside GM2-activator protein and vesicular transport in collecting duct intercalated cells. J. Am. Soc. Nephrol. 10:435-443.

24. Reiser, J., Kriz, W., Kretzler, M., and Mundel, P. 2000. The glomerular slit diaphragm is a modified adherens junction. J. Am. Soc. Nephrol. 11:1-8.

25. Snyers, L., Umlauf, E., and Prohaska, R. 1999. Association of stomatin with lipid-protein complexes in the plasma membrane and the endocytic compartment. Eur. J. Cell Biol. 78:802-812.

26. Brown, D.A., and Rose, J.K. 1992. Sorting of GPI-anchored proteins to glycolipid-enriched membrane subdomains during transport to the apical cell surface. Cell. 68:533-544.

27. Simons, M., et al. 2001. Involvement of lipid rafts in nephrin phosphorylation and organization of the glomerular slit diaphragm. Am. J. Pathol. 159:1069-1077.
28. Engelman, J.A., Zhang, X.L., Razani, B., Pestell, R.G., and Lisanti, M.P. 1999. p42/44 MAP kinase-dependent and -independent signaling pathways regulate caveolin-1 gene expression. Activation of Ras-MAP kinase and protein kinase A signaling cascades transcriptionally down-regulates caveolin-1 promoter activity. J. Biol. Chem. 274:32333-32341.

29. Ruotsalainen, V., et al. 1999. Nephrin is specifically located at the slit diaphragm of glomerular podocytes. Proc. Natl. Acad. Sci. USA. 96:7962-7967.

30. Holthofer, H., et al. 1999. Nephrin localizes at the podocyte filtration slit area and is characteristically spliced in the human kidney. Am. J. Pathol. 155:1681-1687.

31. Inoue, T., et al. 2001. FAT is a component of glomerular slit diaphragms. Kidney Int. 59:1003-1012.

32. Schnabel, E., Anderson, J.M., and Farquhar, M.G. 1990. The tight junction protein ZO-1 is concentrated along slit diaphragms of the glomerular epithelium. J. Cell Biol. 111:1255-1263.

33. Piepenhagen, P.A., and Nelson, W.J. 1995. Differential expression of cellcell and cell-substratum adhesion proteins along the kidney nephron. Am. J. Physiol. 269:C1433-C1449.

34. Kawachi, H., et al. 2000. Cloning of rat nephrin: expression in developing glomeruli and in proteinuric states. Kidney Int. 57:1949-1961.

35. Kurihara, H., Anderson, J.M., and Farquhar, M.G. 1995. Increased Tyr phosphorylation of $\mathrm{ZO}-1$ during modification of tight junctions between glomerular foot processes. Am. J. Physiol. 268:F514-F524.

36. Seiler, M.W., Venkatachalam, M.A., and Cotran, R.S. 1975. Glomerular epithelium: structural alterations induced by polycations. Science. 189:390-393

37. Caulfield, J.P., Reid, J.J., and Farquhar, M.G. 1976. Alterations of the glomerular epithelium in acute aminonucleoside nephrosis. Evidence for formation of occluding junctions and epithelial cell detachment. Lab. Invest. 34:43-59.

38. Li, C., Ruotsalainen, V., Tryggvason, K., Shaw, A.S., and Miner, J.H. 2000. CD2AP is expressed with nephrin in developing podocytes and is found widely in mature kidney and elsewhere. Am. J. Physiol. Renal Physiol. 279:F785-F792.

39. Scheiffele, P., et al. 1998. Caveolin-1 and -2 in the exocytic pathway of MDCK cells. J. Cell Biol. 140:795-806.

40. Salzer, U., Kubicek, M., and Prohaska, R. 1999. Isolation, molecular characterization, and tissue-specific expression of ECP-51 and ECP-54 (TIP49), two homologous, interacting erythroid cytosolic proteins. Biochim. Biophys. Acta. 1446:365-370.

41. Verkade, P., Harder, T., Lafont, F., and Simons, K. 2000. Induction of caveolae in the apical plasma membrane of Madin-Darby canine kidney cells. J. Cell Biol. 148:727-739.

42. Shih, N.Y., et al. 2001. CD2AP localizes to the slit diaphragm and binds to nephrin via a novel C-terminal domain. Am. J. Pathol. In press.

43. Shaw, A.S., and Miner, J.H. 2001. CD2-associated protein and the kidney. Curr. Opin. Nephrol. Hypertens. 10:19-22. 